\title{
El comportamiento relacionado con la información en la literatura en lengua hispana
}

\author{
Information behaviour in the Spanish academic literature
}

\author{
Michela MONTESI \\ Facultad de Ciencias de la Documentación, Universidad Complutense de Madrid, España \\ mmontesi@pdi.ucm.es
}

\begin{abstract}
Resumen
Se revisa la literatura publicada en español sobre comportamiento relacionado con la información. Para complementar estudios anteriores sobre estudios de usuarios, necesidades y uso de la información, expresiones más comunes en la literatura en lengua hispana, adoptamos el punto de vista de la literatura americana que define el information behavior como la totalidad de los comportamientos humanos relacionados con la información. Los 121 documentos recuperados buscando en 4 bases de datos las expresiones "comportamiento informativo", "comportamiento informacional" y "conducta informativa", permiten aislar varias diferencias. En particular, se destacan diferencias conceptuales así como de énfasis, pues la investigación sobre comportamiento de información en español suele relacionarse con la alfabetización informacional y la formación de usuarios.
\end{abstract}

Palabras clave: Comportamiento informativo. Comportamiento informacional. Conducta informativa. Revisión de la literatura. Alfabetización informacional.

\section{Introducción}

En las últimas décadas la gran popularidad de las tecnologías de la información y comunicación ha causado el surgimiento o la consolidación de nuevas especialidades dentro de la documentación centradas en las personas consumidoras de información. El interés por las necesidades de información y más en general por el comportamiento en relación con la información es tan antiguo como la propia documentación, y constituye una de las dos orientaciones dentro de la disciplina al lado de la que se ocupa de técnicas, sistemas y tecnología (Saracevic 2010 , p. 2570). A pesar de estar presente entre las prioridades de la disciplina desde sus albores, es a partir de los años setenta cuando el foco de interés pasa realmente de los sistemas de acceso a la información y las fuentes de información a las personas (Dervin y Nilan 1986). Y según Case, autor de la primera monografía dedicada en exclusiva al tema (Case 2002), sería solo después de Internet cuando la investigación sobre comportamiento e informa-

\begin{abstract}
We review the literature on information behavior published in Spanish. In order to complement previous studies on user studies, information needs and information use, the most common expressions in the literature in Spanish, we adopt the point of view of the literature published in English that defines information behavior as the totality of behaviors related to information. The 121 documents retrieved looking in 4 databases for the phrases "comportamiento informativo", "comportamiento informacional", and "conducta informativa", allow to highlight several differences between the literature published in Spanish and the literature published in English. Particularly, conceptual differences are noticeable as well as a tendency to relate information behavior research to information literacy.
\end{abstract}

Keywords: Information behaviour. Spanish research. Literature review. Information literacy.

ción, que estudiaba básicamente sistemas y lugares de la búsqueda de información, pasa a centrarse de verdad en el comportamiento humano propiamente dicho (Case 2007, p. 6-7).

Case (2007, p. 5) define el comportamiento relacionado con la información de la siguiente forma:

[...] abarca la búsqueda de información y todos los otros comportamientos involuntarios o pasivos (como ojear o encontrar información), así como todos los comportamientos intencionales que no involucran la búsqueda como el hecho de evitar activamente la información.

Fisher et al., introduciendo la monografía Theories of Information Behavior, conceptualizan el comportamiento relacionado con la información como la forma en la que "las personas necesitan, buscan, gestionan, dan, y utilizan la información en diferentes contextos" (2005, p. $\mathrm{xix})$.

Un intenso debate en la década de los noventa produjo la consolidación de la disciplina así 
como de la propia expresión "information behavior" (Pettigrew y McKechnie 2001, p. 44-45). La literatura americana actual entiende el comportamiento relacionado con la información como un proceso del que forman parte otros subprocesos como, entre ellos, el de "terrenos de la información" (information grounds) -que para otros no sería otra cosa que el contexto-, búsqueda de información (information seeking), búsqueda en sistemas de información (information searching), organización de la información (information organizing), uso de la información (information using), etc. (Spink 2010, p. 65-68).

Queda patente que en la literatura americana cuando hablamos de comportamiento e información entendemos algo más que estudios de usuarios y de necesidades informativas. El comportamiento relacionado con la información abarca todo fenómeno relacionado con necesidades de información, búsqueda de información, modalidades de organización de la información (Spink y Cole 2004), así como todo otro comportamiento relacionado con la información como el propio hecho de eludir la información.

Pero, ¿cuál es la situación de estos estudios en España? González Teruel y Abad García (2007) revisan la literatura publicada en España en el periodo 1990-2004 restringiendo su ámbito exclusivamente a las necesidades y usos de información. En un estudio anterior, González Teruel (2005, p. 34-35) explica que las denominaciones de "estudios de usuarios" o "estudios de necesidades y usos de la información" son más populares en España que la expresión "conducta informativa". Buscando indicios de un cambio de tendencia en la literatura española parecido al ocurrido en ámbito anglosajón, de una perspectiva centrada en el sistema a una centrada en la persona, las dos autoras concluyen que el área de necesidades y usos de información en España aún no es un área consolidada. Prueba de ello serían el número reducido de publicaciones (después de su exhaustiva búsqueda solo consiguieron encontrar 123 publicaciones relevantes), la tendencia a presentar los estudios sobre necesidades y uso de la información en conferencias más que en revistas científicas, y la ausencia de estudios teóricos sobre el tema. Resultados parecidos obtiene Villaseñor Rodríguez (2009) después de analizar una muestra de 36 artículos y ponencias publicados en España en el periodo 2000-08 sobre estudios de usuarios. Constata, además, que en lo que concierne a la autoría predominan los profesionales de la información, y que su objetivo es conocer mejor el uso o las necesidades y hábitos de información. Asimismo, el $41,66 \%$ de estos estudios pretende medir la satisfacción del usuario respecto a un centro, un servicio o una fuente de información, tratándose de trabajos de naturaleza fundamentalmente aplicada. Villaseñor Rodríguez (2010a), por otro lado, encontraba solo 26 trabajos publicados en España desde 1994 hasta 2009 que trataban el tema desde un punto de vista teórico. Todos se habían publicado tras la aparición del Manual de estudios de usuarios de Elías Sanz Casado (Sanz Casado, 1994), y se centraban principalmente en aspectos metodológicos y técnicas de recogida de datos.

Precisamente la aplicación de un método de investigación a un objeto claro de estudio, el usuario, permite afirmar que los estudios de usuarios reúnen los requisitos para ser una línea de investigación independiente (Villaseñor Rodríguez, 2010b). Sin embargo, a juicio de la autora, dicha línea de investigación no goza del necesario reconocimiento en Biblioteconomía y Documentación (BD), como sería evidente de su escasa presencia en los planes de estudio universitarios. Al respecto Lascurain-Sánchez (2007, p. 154) comenta que "el interés por los Estudios de Usuarios y su evolución metodológica y conceptual no se corresponden en nuestro país con su presencia en los currícula universitarios", pues al revisar los planes de estudio de las titulaciones de BD de las universidades públicas españolas en el curso 2006-07, la asignatura de Estudios de Usuarios aparecía como obligatoria solo en dos universidades, la Carlos III de Madrid y la de Granada.

Con la intención de complementar los estudios reseñados arriba, el presente trabajo pretende ver la investigación realizada en España o en español sobre estudios de usuarios desde el punto de vista de la literatura americana. Por esta razón optamos por analizar la investigación sobre "comportamiento informativo", "comportamiento informacional" y "conducta informativa", expresiones con las cuales se puede traducir el concepto de "information behavior". De esta forma, creemos que se pueden iluminar aspectos que las revisiones bibliográficas anteriores, concretamente las de González-Teruel y Abad-García (2007) y Villaseñor Rodríguez (2009), no han llegado a cubrir, pues se han centrado en los estudios de usuario. Entre las otras pretendemos proponer una respuesta para las siguientes preguntas: ¿En qué contextos aparecen las expresiones "comportamiento informativo", "comportamiento informacional", y "conducta informativa"? ¿Cómo de populares son? ¿De qué trata la investigación sobre comportamiento informativo, comportamiento informacional y conducta informativa? ¿Cómo se 
relaciona con la literatura americana sobre el mismo tema?

\section{Cuestiones metodológicas y de procedimiento}

Para contestar a las preguntas anteriores procedimos de la siguiente forma. En primer lugar aislamos unas obras de referencia, lo más recientes posible, sobre comportamiento relacionado con la información en lengua inglesa. Concretamente, se trata de las dos monografías de Donald O. Case antes mencionadas, la Encyclopedia of Information Science coordenada por Bates y Maack y publicada en 2009, y la obra coordenada por Fischer, Erdelez y Mckechnie (2005), que reúne 72 entradas redactadas por sendos autores sobre teorías de information behavior. La última edición de la monografía de Case (Case 2007) reúne más de mil referencias bibliográficas. Por otro lado, la de Fischer et al. (2005) se publicó tras el Tercer Simposio del Special Interest Group on Information Seeking and Use (SIG USE) de ASIST en 2003. Los participantes en el simposio son también los autores de las 72 entradas. Las obras mencionadas, en nuestra opinión, permiten afirmar que existe consenso en la literatura americana acerca de los conceptos fundamentales del área de estudio, pues estos trabajos de referencia presentan un alto grado de acuerdo.

Posteriormente localizamos publicaciones en español relacionadas con el tema, buscando los conceptos de "comportamiento informativo" (CIVO en adelante), "comportamiento informacional" (C-AL en adelante), y "conducta informativa" (C-CTA en adelante) en cuatro bases de datos: 1) ISOC de Biblioteconomía y Documentación (ISOC); 2) Library and Information Science Abstracts (LISA); 3) ISI Web of Science (ISI WoS); y 4) Google Scholar (GS). La cobertura y las funcionalidades de estas bases varían influyendo en los resultados que se pueden obtener. Por ejemplo, ISI WoS permite buscar solo en inglés aunque deja indicar el país de la institución de filiación (en este caso España), opción que no admite LISA. En cuanto a GS, hay que destacar que el buscador indiza, aparte índices de publicaciones científicas y bases de datos especializadas, el texto completo de aquellos documentos que se encuentran en acceso abierto, y consecuentemente devuelve con el resultado incluso aquellos que no mencionan el concepto en el título o en los descriptores. Es multidisciplinar y además recupera literatura gris, sitios Web y documentos en autopublicación que no constan en las otras bases de datos. Por otro lado, trabajando de forma automática sin intervención humana, no ofrece los mismos niveles de precisión de las otras bases de datos.

Inicialmente optamos por buscar sencillamente las expresiones C-IVO, C-AL, y C-CTA en lugar de "comportamiento en la búsqueda de información" o "comportamiento informativo" + "búsqueda de información" u otros descriptores relacionados como hacen González-Teruel y AbadGarcía (2007). Estas dos autoras emplean una estrategia de búsqueda amplia incluyendo todos los términos que puedan caracterizar algún aspecto del proceso de búsqueda de información, como user studies, information needs, information-seeking behavior, etc., pero su objetivo es diferente y además se encuentran con mucho ruido. Posteriormente se ven obligadas a eliminar un $70 \%$ de los resultados obtenidos en una base de datos y a seleccionar artículos relevantes de una muestra de 2905 revistas, con un mayor riesgo de error al revisar manualmente tanto volumen de revistas. Así que decidimos buscar sencillamente las tres expresiones de arriba. Finalmente, decidimos recuperar solo documentos publicados a partir de 1994, ya que Villaseñor Rodríguez en un estudio anterior constataba que la gran mayoría de estudios de usuarios se habían publicado a partir de entonces (Villaseñor Rodríguez, 2010a).

Los resultados obtenidos de las cuatro bases de datos en varias búsquedas realizadas en el mes de mayo de 2011 están reflejados en la Tabla I.

\begin{tabular}{lccc}
\hline Base de datos & C-IVO & C-AL & C-CTA \\
\hline ISOC & 1 & 5 & 2 \\
\hline LISA & 4 & -- & 1 \\
& & & \\
\hline ISI WoS & 5 & -- & -- \\
\hline GS & 49 & 49 & 17 \\
\hline Total & 59 & 54 & 20 \\
\hline
\end{tabular}

Tabla I. Número de documentos recuperados en las bases de datos

Todos los documentos recuperados en ISOC, LISA, y ISI WoS se consideraron relevantes, aunque se descartó un documento de LISA por estar escrito en portugués. En GS, en cambio, fue necesario depurar la muestra por varias razones. Muchos de los registros recuperados son duplicados o repeticiones; algunos documentos están escritos en otros idiomas o se sitúan claramente en otras disciplinas como el periodismo; otros utilizan las expresiones bus- 
cadas con otros significados o en alguna referencia bibliográfica. Finalmente, decidimos excluir ciertos tipos de documentos como presentaciones o reseñas de libros, puesto que o bien están pensadas para otros fines, o bien no presentan de forma suficientemente clara la postura del autor acerca de los temas objeto de estudio.

\section{Resultados}

En total, de los 18 documentos recuperados a través de ISOC, LISA e ISI WoS, 12 se encuentran con GS también. En lo específico, solo dos de los documentos recuperados a través de ISOC no aparecen en la muestra de GS. Los resultados obtenidos a través de LISA se repiten por completo en las muestras de GS. Finalmente, de las cinco publicaciones recuperadas por el ISI WoS solo una coincide con LISA y GS, mientras que las otras son trabajos empíricos de autores afiliados con instituciones españolas y que están escribiendo en revistas internacionales en lengua inglesa. Tres de éstos tratan de comportamiento de información en entornos académicos. Borrego y Urbano (2007) y Ollé y Borrego (2010) analizan el impacto de las revistas electrónicas en el comportamiento de investigadores y docentes, mientras que Pinto et al. (2010) analizan el comportamiento de usuarios de una biblioteca universitaria en relación a la calidad de servicios de la misma. Ferrán et al. (2007) analizan el comportamiento de usuarios de plataformas para la docencia virtual para mejorar los metadatos correspondientes a objetos de aprendizaje.

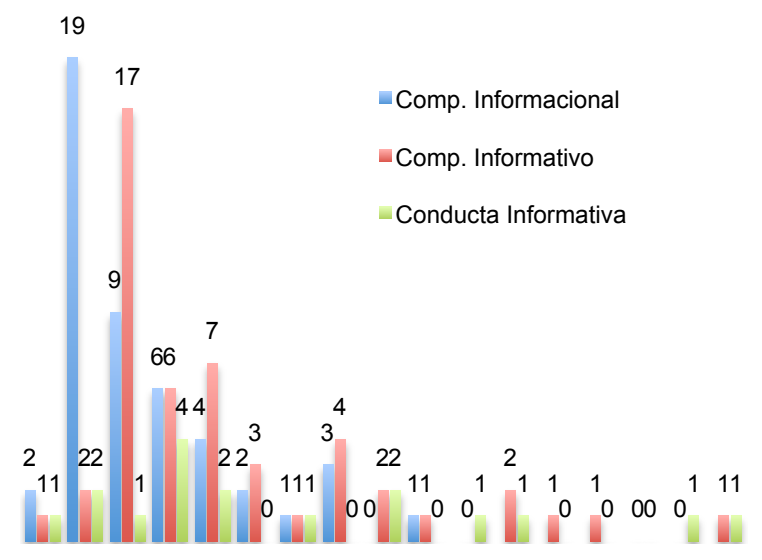

Gráfico 1. Número de documentos en los que aparecen las expresiones C-IVO, C-AL, C-CTA. 1995-2011.

GS fue la base de datos que devolvió el mayor número de resultados en las búsquedas con las tres expresiones. Significativamente, el solapamiento entre las tres muestras de resultados es mínimo, es decir que pocos documentos se recuperan por dos o más de las expresiones buscadas. C-AL comparte un documento con $\mathrm{C}$ IVO y dos con C-CTA, mientras que C-CTA y CIVO solo comparten un registro. Esto apunta a que las tres expresiones aíslan tres muestras distintas de contribuciones. En el Gráfico 1, notamos que si la expresión C-AL no aparece hasta 2002, en la actualidad es la que aparece en un mayor número de documentos. C-CTA, por otro lado, aparece a mediados de los noventa pero nunca consigue mucha popularidad comparada con las otras dos expresiones.

Analizando los documentos en más detalle, nos encontramos con que en la muestra de C-IVO, destaca la presencia de contribuciones del autor Juan José Calva González —nada menos que 13, algunas de las cuales son ediciones de obras colectivas, como Calva González (2009)_, o de estudiosos mejicanos que adoptan su marco teórico. En la página Web del Seminario de Usuarios de la Información que este investigador viene organizando desde 2004 en la Universidad Nacional Autónoma de México (Calva González 2010), se exponen los principios teóricos que comparten los participantes en el seminario:

El soporte y unión que sostiene este seminario y el trabajo conjunto de los integrantes del mismo [...] es la concepción de que el fenómeno de las necesidades de información es un ciclo que se conforma de tres fases substanciales: las necesidades de información, el comportamiento informativo y la satisfacción de dichas necesidades.

Entre las acciones que el seminario se propone emprender está la aclaración de conceptos y términos, entre los cuales destaca por su ausencia la propia expresión de C-IVO, aunque sí aparece en frases como "Manifestación contra comportamiento informativo", o "patrones de comportamiento informativo". Por otro lado, destaca también la presencia de terminología propia de la recuperación de información como pertinencia, relevancia, y precisión, así como de objetos informativos como las fuentes de información, primarias, secundarias, terciarias..., que pueden apuntar hacia un enfoque en el sistema más que en las personas. De hecho, Calva González (1998) defiende la necesidad de conocer las necesidades de información de los usuarios para la automatización de unidades de información y la construcción de sistemas de recuperación de información. Este requisito aparece también en otros autores de la muestra, como por ejemplo Negrete Gutiérrez (1999), que habla de conocer mejor el C-IVO de las comuni- 
dades de referencia de las bibliotecas universitarias ante el nuevo paradigma tecnológico informacional y para el desarrollo de las colecciones.

El modelo NEIN (NEcesidades de INformación), que resume la visión de Calva González, se encuentra esbozado en varias contribuciones de este autor (incluidas Calva González, 2004, 2007). De las tres fases discernibles en el fenómeno de la necesidad de información, la satisfacción, concepto ausente en la literatura americana, sería "el restablecimiento del equilibrio del sujeto con su medio circunstante, a partir de la desaparición de las carencias de conocimiento e información" (Calva González, 2009 , p. 3-4). Esta concepción teórica se encuentra también en otros documentos de la muestra como el de Tannuri et al. (2007). Aunque según el modelo NEIN, la satisfacción de las necesidades de información se puede conseguir por dos caminos, o bien a través de la experiencia personal o bien a través de las "unidades de información", la investigación -que se basa en la formulación teórica de Calva González y que recuperamos en su gran mayoría por G- se centra exclusivamente en las unidades de información. Muchos estudios empíricos, utilizando el referente teórico de Calva González, analizan las necesidades de información en comunidades concretas. El concepto de "comunidad" aparece en los títulos de los cinco Seminarios de Usuarios de la Información celebrados desde 2004. Al centrarse en una comunidad, se define a los usuarios por su ocupación, o sector social como las comunidades indígenas (Ramírez Velázquez, 2011) o los adolescentes (Calva González, 2006). Una de las "comunidades" más estudiada son los profesores e investigadores. Muchos estudios describen fuentes y recursos utilizados como rasgo principal del C-IVO de este colectivo, como por ejemplo, por citar a algunos, los de Calva González (2003), García Velarde (2008) —quien analiza el C-IVO de investigadores de las ciencias sociales de una universidad mejicana a través de las referencias incluidas en las tesis de postgrado-, Santos Rosas y Calva González (2008) y Guevara Villanueva (2009) —quienes estudian la comunidad de los matemáticos-, y Santos Rosas (2009) quien analiza la comunidad de los investigadores en geografía.

Con C-IVO, al lado de los numerosos estudios de Calva González o inspirados en sus teorías, muchos de los cuales son de naturaleza empírica, GS nos devuelve además otros de talante más teórico, que tratan de la disciplina de la BD y de ramas o líneas de investigación dentro de ésta, como el de Vega Almeida et al. (2009), por ejemplo, de Mancipe Flechas et al. (2009), o de Fernández Molina y Moya Anegón (2002), quienes repasan la historia de la BD a través de tres paradigmas: positivista, cognitivo y social. Martínez Gutiérrez (2003) emplea el concepto de CIVO para definir otro, es decir el de consumo de información, destacando por ser uno de los pocos estudios donde se utiliza el concepto de C-IVO en relación al uso de las TICs.

Finalmente hay una serie de estudios donde el C-IVO se trata en relación a temáticas de formación de usuarios o de alfabetización informacional (González Harmón y Limón González 2005; Repanovici y Landoy, 2007; Balderas Chávez y Martínez Moreno, 2009). García Rivadulla (2009) entrevista a un experto en comportamientos informativos de adolescentes y niños con el objetivo de conocer las competencias esenciales de una persona alfabetizada.

Esta asociación entre comportamiento y alfabetización informacional o formación de usuarios aparece de forma rotunda en la muestra que GS nos devuelve cuando buscamos C-AL, pues la encontramos en 26 de los 49 documentos recuperados. Destaca entre los resultados obtenidos el trabajo de Uribe desarrollado a partir de su tesis doctoral (Uribe Tirado, 2008), quien afirma que el desarrollo de la ALFIN depende de saber relacionarla con modelos teóricos de C-AL entendido como en González Teruel (2005) y en la literatura americana, es decir: "la totalidad de comportamientos humanos en relación con los recursos y canales de información" (Uribe Tirado, 2008, p. 14). Según Uribe Tirado (2010), el conocimiento de los comportamientos informacionales de los usuarios implicados en programas de ALFIN permite dar el paso de la formación de usuarios tradicional a una formación como medio para el aprendizaje permanente y la generación de conocimientos. Comparten esta visión otros autores de la muestra como Puertas y Pinto (2009) o Diaz (2010).

Güell Guillén y Casals Parladé (2009), Serrat Brustenga y Sunyer Lázaro (2008), y Novoa et al. (2008), en cambio, mencionan el concepto de $\mathrm{C}-\mathrm{AL}$ en relación a servicios de bibliotecas universitarias y CRAIS, todos en clave teórica. En conexión a la formación de usuarios, se pueden mencionar a Nuñez Paula (2004) y a Martí-Lahera (2004), quien hace referencia explícita a teorías de C-AL como referente teórico de la formación de usuarios. Significativamente, cuando el concepto de comportamiento se adapta a la formación y a la ALFIN, el adjetivo informacional sustituye a informativo en otras expresiones: habilidades informacionales, necesidades informacionales... (Serrat Brustenga y Sunyer Lázaro, 2008). 
En esta muestra, el vínculo entre el C-AL y las TICs destaca más que en las otras dos. Mientras en C-IVO, Internet aparecía a menudo como una fuente de información más, cuando se estudia el $\mathrm{C}-\mathrm{AL}$, se percibe como necesario realizar un diagnóstico del nivel de las competencias informacionales de los usuarios de referencia (De la Cruz Santos, 2005), o definir perfiles de usuarios con base en sus habilidades en la búsqueda y gestión de la información para la adquisición de competencias informacionales y mejora de entornos virtuales (Ferrán Ferrer y Pérez Montoro, 2009).

No siempre el uso de la expresión C-AL coincide con un enfoque explícito en la ALFIN, pues pueden intervenir factores lingüísticos. Por ejemplo, la expresión "information behavior" que aparece en la traducción del Informe Ciber de la British Library "Information behaviour of the researcher of the future" (Moreno Pascual, 2008), publicado por la revista Anales de Documentación, se traduce con C-AL. A veces en un mismo texto traducido del inglés $u$ otro idioma, C-IVO y C-AL se alternan, como en Tannuri et al. (2007) o Nghiem (2010).

Por otro lado, factores disciplinarios pueden intervenir en la elección de la expresión C-AL, ya que es ésta la expresión favorita en las 7 contribuciones de la muestra que tratan de la información en ámbito organizativo y empresarial como, por citar a algunos, Rodríguez Cruz y Pinto Molina (2010) y Beckman Cavalcante y Pomin Valentim (2010).

Finalmente por lo que concierne a la expresión C-CTA, aunque se trate de la más antigua, sigue apareciendo en contribuciones recientes, como Ollé Castellá (2010), quien utiliza la expresión en una tesis sobre el impacto de las revistas electrónicas en el comportamiento de profesores e investigadores de universidades catalanas, o Montesi (2008), quien menciona el concepto en una revisión de la literatura sobre recuperación de información según el concepto de "género Web".

No emerge para C-CTA un marco teórico claro como para las otras dos expresiones, y parece más una opción debida a la indeterminación terminológica. Hay autores expertos en estudios de usuarios que lo emplean en alternativa a una de las otras dos expresiones como Villaseñor Rodríguez (2008) o González-Teruel (2010). Hernández Salazar et al. (2007) hacen referencia al caos terminológico relativo a lo que denominan "proceso de comportamiento en la búsqueda de información" que podría solucionarse, en su visión, analizando tres modelos de comportamiento relacionado con la información, concretamente los de Krikelas, Kuhlthau, y Wilson. Analizados estos tres modelos concluyen que comportamiento es sinónimo de conducta. En la monografía de González Teruel (2010), CCTA aparece numerosas veces, aunque la autora recientemente se ha decantado por la expresión $\mathrm{C}-\mathrm{AL}$.

Quizás la supervivencia y relativo éxito de la expresión se deba a un famoso estudio de Romanos de Tiratel (2000) sobre los hábitos informativos de investigadores de las ciencias sociales y humanidades en Argentina, donde la autora, tras realizar un encuesta, concluía que la conducta informativa de estos investigadores coincidía en gran medida con la de sus correspondientes anglosajones. Este estudio ha inspirado a otros autores argentinos, en su mayoría bibliotecarios, a realizar encuestas parecidas, como (Gómez 2001; Münster 2003; Domínguez 2010).

\section{Discusión}

Buscando en cuatro bases de datos las expresiones C-IVO, C-AL, y C-CTA, que consideramos las correspondientes de una consolidada expresión inglesa como information behavior, encontramos que se nos devuelven un total de 121 documentos (excluyendo los solapamientos, es decir, los documentos que se recuperan utilizando más de una expresión) publicados a partir de 1994. La gran mayoría de las publicaciones encontradas se pueden localizar gracias a GS, mientras que no aparecen en bases de datos especializadas como ISOC de Biblioteconomía y Documentación. Este hecho combinado con una tendencia al alza en el número de publicaciones según refleja el Gráfico 1, puede interpretarse como que el área es joven y aun marginal con respecto a otras áreas consolidadas dentro de la BD. De hecho muchos autores son profesionales y sus publicaciones pueden encontrar menor aceptación en las publicaciones académicas. Por otro lado, es evidente la diversidad terminológica del campo de investigación, a su vez signo de inmadurez. Es muy significativo que los autores más productivos y conscientes de esta diversidad terminológica como Villaseñor Rodríguez, González Teruel, o la propia Hernández Salazar —quien colabora además con estudiosos anglosajones (Hernández Salazar et al. 2010)— sean precisamente quienes más oscilan entre una expresión u otra, empleando a veces C-IVO, otras C-AL, otras CCTA. En este sentido percibimos una primera diferencia con la literatura americana que, a pesar de caracterizarse por numerosas teorías y marcos de estudio, suele coincidir sobre la terminología de base, según queda reflejado en la 
Encyclopedia of Information Science coordinada por Bates y Maack o en la recopilación de 72 teorías sobre information behavior que realizan Fischer et al. (2005).

También se pueden destacar diferencias de orden conceptual especialmente donde la reflexión teórica es más abundante como en la teorización del fenómeno de las necesidades de información de Calva González. Cuando tomamos el concepto de C-IVO definido por Calva González y adoptado por otros estudiosos, vemos que mientras que en la literatura americana el information behavior engloba todo comportamiento humano relacionado con la información, Calva González lo entiende más como "comportamiento en la búsqueda de información", pues en su visión es el comportamiento desencadenado por una necesidad de información. El propio énfasis de esta corriente en las necesidades de información choca con las tendencias actuales de la escuela americana. Explica Saracevic (2010) que el concepto de necesidades de información ha perdido protagonismo por ser un concepto nebuloso, una experiencia subjetiva en la mente de alguien que no es susceptible de observación, y, además, por ignorar otros aspectos de la realidad. Sin embargo, sigue presente en los modelos de recuperación de información como el origen de las preguntas que se ponen a un sistema de recuperación. Más arriba interpretábamos este hecho como signo de que la literatura sobre C-IVO según la entiende Calva González y los numerosos estudiosos que lo respaldan sigue enfocada más en el sistema que en las personas. Otra diferencia destacable sería la concepción del "fenómeno de las necesidades de información" como un proceso lineal, en el que se diferencian tres fases consecutivas: necesidad, comportamiento informativo, satisfacción. En la literatura americana, desde la publicación de Foster (2004), los procesos de búsqueda de información se conciben cada vez más como no lineales.

Más en general, en la literatura en lengua española notamos cierto énfasis en conceptos menos enfatizados o ausentes en la americana, como satisfacción y calidad, o comunidades de usuarios. En lugar de satisfacción, en la literatura americana se suele hablar de elementos emocionales y afectivos involucrados en la relación entre el individuo y la información (Kulthau, 2004). La importancia del concepto de satisfacción se puede achacar a la influencia de la literatura de ámbito empresarial y organizativo que tiene una parte destacable, además, en la muestra que GS devuelve al buscar C-AL. En cuanto al concepto de comunidades, ya reseñamos más arriba algunos de los estudios empí- ricos realizados en el ámbito de comunidades específicas. Naumer y Fischer (2010) explican que en la historia de la investigación sobre necesidades de información, el foco en cuestiones sociales y de comunidades aparece alrededor de los años 60-70, con el objetivo de aislar las necesidades de información de amplias poblaciones. Hoy en día la literatura americana prefiere el concepto de contexto al de comunidad.

Además de las diferencias conceptuales que acabamos de reseñar, otro de los datos más significativos de la revisión es la asociación entre el comportamiento con relación a la información y la alfabetización informacional y/o la formación de usuarios, especialmente en las bibliotecas universitarias. Este aspecto no emerge de forma clara en revisiones anteriores como las de González-Teruel y Abad-García (2007) o Villaseñor Rodríguez (2009), ya que éstas se centraban en estudios de usuarios, necesidades y uso de la información. Aunque esta asociación es algo propio de la literatura americana también es notable su presencia en las muestras analizadas y justifica la tendencia al alza de las contribuciones sobre C-IVO y CAL. Confirma además un aspecto destacado por Villaseñor Rodríguez (2009) de la literatura sobre estudios de usuarios, es decir, su conexión con la práctica y los profesionales, pues muchos autores de trabajos sobre C-AL son bibliotecarios. Además con mucha frecuencia en estos estudios se mencionan las TICs y su papel en el universo informativo de las personas que se pretende "alfabetizar" (Ferrán Ferrer y Pérez Montoro, 2009). Si queda clara la necesidad de conocer el comportamiento de las personas para emprender planes de ALFIN destinados a estas, muchas veces los estudios que se realizan, tanto empíricos como de reflexión, carecen de unas bases teóricas fuertes y los referentes teóricos son escasos. La razón puede encontrarse no solo en la escasez de estudios teóricos sobre comportamientos de información y estudios de usuarios, sino en la propia naturaleza de los existentes. Muchos se centran en la disciplina de BD en su totalidad, subrayando el lado humano que los estudios de usuarios aportan a la BD. Por otro lado, los trabajos teóricos sobre estudios de usuarios insisten a menudo en cuestiones de tipo metodológico afines a la bibliometría como en Sanz Casado (1994) o Sanz Casado y Martín Moreno (1997), que pueden resultar de difícil aplicación o lejanas del entorno "humano" de quienes trabajan en alfabetización informacional y formación de usuarios. En fin, aunque existen excepciones recientes como González Teruel (2010), es posible que los pocos trabajos de naturaleza teórica no ofrezcan claves de fácil aplicación para la inter- 
pretación de la realidad de quienes realizan estudios empíricos con personas, a menudo los propios bibliotecarios.

\section{Conclusiones}

Para concluir, creemos importante destacar la tendencia al alza de la literatura sobre los comportamientos en relación a la información, especialmente de trabajos que adoptan el concepto de $\mathrm{C}-\mathrm{AL}$, que además parece tener mayores probabilidades de consolidarse como equivalente de information behavior según lo concibe la literatura americana. Asimismo, el énfasis de la literatura en español en la ALFIN, la relación con la literatura de ámbito empresarial y organizativo, así como las diferencias conceptuales con la literatura americana, permiten afirmar que los estudios en español sobre comportamiento relacionado con la información presentan unas características e identidad propias. Finalmente, estos estudios apuntan a una brecha entre teoría y práctica que pide la necesaria atención por parte de los estudiosos. Si por un lado muchos estudios con usuarios de información se realizan por profesionales, éstos carecen a menudo de las bases teóricas y metodológicas apropiadas. Sin embargo, la literatura científica parece insuficiente y aún incapaz de proporcionar el necesario referente teórico.

\section{Referencias}

Arribas Urrutia, A. (2009). Informar y comunicar a través de las nuevas tecnologías para crear cultura corporativa en la empresa. // Palabra Clave. 8, 135-172.

Balderas Chávez, F.A.; R.M. Martínez Moreno. (2009). Adolescentes e información. // Tercer encuentro de estudiantes de bibliotecología y ciencias de la información. San Nicolás de los Garza, Nuevo León. Universidad Autónoma de Nuevo León, 2009.

Bates, M. J.; M. N Maack. (2009). Encyclopedia of library and information sciences. Boca Raton, FL, USA: CRC Press, Inc., 2009.

Beckman Cavalcante, L. F.B.; Pomin Valentim, M. L. (2010). Cultura y comportamiento informacional en ambientes empresariales y el proceso de inteligencia competitiva. // Biblios: Revista de Bibliotecología y Ciencias de la Información. 39, 1-10.

Borrego, A.; Urbano, C. (2007). Analysis of the behaviour of the users of a package of electronic journals in the field of chemistry. // Journal of Documentation. 63:2, 243-258.

Calva González, J. J. (2003). Las necesidades de información de los investigadores del área de Humanidades y Ciencias Sociales. // Revista general de información y documentación. 13:2, 155-180.

Calva González, J. J. (2004). Las necesidades de información: fundamentos teóricos y métodos. México: Universidad Nacional Autónoma de México, Centro Universitario de Investigaciones bibliotecológicas, 2004.

Calva González, J. J. (2006). Información y adolescencia: búsqueda de información por los adolescentes. // Memoria del Tercer Seminario Hispano-Mexicano de investi- gación en bibliotecología y documentación 29 al 31 de marzo de 2006. 73-89.

Calva González, J.J. (2007). Los estudios sobre las necesidades de información: acercamiento a los últimos 25 años en México y América Latina. // Martínez Arellano, F.; Calva González, J. J. (editores) 2007. Memoria del XXIV Coloquio de Investigación bibliotecológica y de la Información. Veinticinco años de investigación en bibliotecología y estudios de la información en México. Centro Universitario de Investigaciones Bibliotecológicas. UNAM. 2007, p. 87.

Calva González, J. J. (2009). Satisfacción de usuarios: La investigación sobre necesidades de información. // Cuadernos de Investigación. 11. México: UNAM, Centro Universitario de Investigación Bibliotecológica: Universidad Nacional Autónoma de Mexico, 2009.

Calva González, J. J., (ed.) (2009). La investigación sobre las necesidades de información en diferentes comunidades. // Memoria del III Seminario de Usuarios de la Información. Vol. 12. Cuadernos de Investigación. México: UNAM, Centro Universitario de Investigación Bibliotecológica. (2009).

Calva González, J. J. (2010). Usuarios de la Información. Seminario de Investigación Permanente. Seminario de usuarios de la información: el fenómeno de las necesidades de información en diferentes comunidades. http://cuib.unam.mx/ usuarios/ (consultado 20/06/2011).

Calva González, J. J. (1998). Las necesidades de información del usuario en la automatización de unidades de información. // Revista Biblioteca Universitaria. 1:1, 5-14.

Case, D. O. (2002). Looking for information: a survey of research on information seeking, needs, and behavior. Amsterdam, New York: Academic Press, 2002.

Case, D. O. (2007). Looking for information: A survey of research on information seeking, needs, and behavior. Amsterdam, New York: Academic Press, 2007.

Cruz Santos, de la, I. D.; Martí Lahera, Y. (2005). Experiencias en la proyección de la alfabetización tecnológicoinformacional en una institución cubana. // Acimed 13:3, $1-1$.

Dervin, B.; Nilan, M. (1986). Information needs and uses. // Annual review of information science and technology. 21, 3-33.

Díaz, M. S. (2010). Competencias informacionales en la formación de las BioCiencias en Cuba. Granada: Universidad de Granada y Universidad de La Habana, 2010. Tesis Doctoral.

Domínguez, L. M. (2008). Users, Internet, and the librarian: user study about online resources. // Información, cultura y sociedad. 18 (Enero-Junio 2008), 57-76.

Fernández Molina, J. C.; Moya Anegón, F. (2002). Perspectivas epistemológicas "humanas" en la Documentación. // Revista española de documentación científica. 25:3, 241-253.

Ferrán, N.; Casadesús, J.; Krakowska, M.; Minguillón, J. (2007). Enriching e-learning metadata through digital library usage analysis. // The Electronic Library. 25:2, 148-165.

Ferrán Ferrer, N.; Pérez Montoro, M. (2009). Gestión de la información personal en usuarios avanzados en TIC. EI profesional de la información. 18:4, 365-373.

Fisher, K. E.; Erdelez, S.; Mckechnie, L. (2005). Theories of information behavior. Medford, Ne Jersey: Information Today Inc., 2005

García Rivadulla, S. (2009). Entrevista al Dr. Andrew K. Shenton, especialista en alfabetización informacional. http://alfinuruguay.blogspot.com/2009/11/interview-withandrew-k-shenton.html (consulta 12/07/2011). 
García Velarde, R. (2008). Uso de fuentes de información en las tesis de posgrado del área de las ciencias sociales en la Universidad Autónoma de Yucatán. // Biblioteca Universitaria: Revista de la Dirección General de Bibliotecas de la UNAM. 2:1 (2008) 22-30.

Gómez, N. D. (2001). La conducta informativa de los físicos: un estudio cualitativo de usuarios. // Información, cultura y sociedad. 4 (2008) 23-26.

González Harmon, R.; Limón González, L. (2005). Estudio del perfil, necesidades de información y programa de formación de usuarios: caso Biblioteca de la Facultad de Filosofía y Letras de la UACH. Foro Transfronterizo de Bibliotecas. Chihuahua, Chihuahua (Mexico), 9-11 marzo (2005) 331-344.

González Teruel, A. (2010). La perspectiva del usuario y del sistema en la investigación sobre el comportamiento informacional. // Teoría de la Educación. Educación y Cultura en la Sociedad de la Información. 12:1 (2010) 9-27.

González Teruel, A. (2005). Los estudios de necesidades y usos de la información: fundamentos y perspectivas actuales. Gijón: Trea, 2005.

González-Teruel, A.; Abad-García, M. F. (2007). Information needs and uses: An analysis of the literature published in Spain, 1990-2004. // Library \& information science research. 29:1 (2007) 30-46.

Güell Guillén, C.; Casals Parladé, C. (2009). Els serveis als usuaris en les biblioteques universitàries». // Item: revista de biblioteconomia i documentación. 2009, 50 (2009) 67-87.

Guevara Villanueva, A. (2009). La medición de la satisfacción del científico matemático en el uso de algunas fuentes de información y servicios bibliotecarios. // Calva González, J. J. (coordinador). La investigación sobre las necesidades de información en diferentes comunidades. Memoria del III Seminario de Usuarios de la Información. México D. F.: Universidad Nacional Autónoma de Mexico (2009) 81-107.

Hernández Salazar, P.; Nicholas, D.; Rowland. I. (2010). Acceso y uso de libros electrónicos por comunidades universitarias del Reino Unido. // Revista Interamericana de Bibliotecología. 32:2 (2010) 13-58

Hernández Salazar, P.; Ibáñez Marmolejo, M.; Valdez Angeles, G.; Vilches Malagón, C. (2007). Análisis de modelos de comportamiento en la búsqueda de información. // Ciência da Informaçao. 36:1 (2007) 136-146.

Lascurain-Sánchez, M. L. (2007). Los estudios de usuarios en los planes de estudio de Biblioteconomía y documentación. // Revista general de información y documentación. 17:2 (2007) 151-158

Mancipe Flechas, E. (2009). Ciencia de la Información: Herramientas teóricas para su comprensión como Ciencia Social. // Códice. 4:2 (2009) 33-40.

Martínez Gutiérrez, G. (2003). El consumo de información. // Hipertext. net. 1 (2003). http://www. hipertext. net/web/pag253. htm (12/07/2011).

Martí-Lahera, Y. (2004). ¿Teoría o metateoría? En el dominio usuario. // Ci. Inf. 33:3, (Set. -Dez. 2004), 50-60.

Montesi, M. (2008). Géneros web: líneas de investigación. // El profesional de la información. 17:5 (2008) 551-558.

Moreno Pascual, L. (2008). Informe ciber. comportamiento informacional del investigador del futuro. Traducción. // Anales de Documentación. 11 (2008) 235-258.

Münster, I. (2003). Un estudio de las necesidades de información, hábitos y características de investigadores en Humanidades y Ciencias Sociales. // Información, cultura y sociedad. 8 (2003) 69-84.

Naumer, C. M.; Fischer, K. E. (2009). Information needs. Bates, M. J.; Maack, M. N. (eds. ), Encyclopedia of Information Science. New York: Taylor and Francis, 2009.
Negrete Gutiérrez, M. C. (1999). El impacto del cambio en el desarrollo de colecciones en bibliotecas universitarias. // Scire: representación y organización del conocimiento. 5:1 (1999) 55-64.

Nghiem, H. (2010). Los programas de la Alfabetización Informacional en el contexto de la sociedad de las tecnologías de la información y las perspectivas interculturales. // World Library and Information Congress: 76th IFLA Conference and Assembly. Gothenburg, Suecia, 10-15 de Agosto de 2010.

Núñez Paula, C. I. (2004). Las necesidades de información y formación: perspectivas socio-psicológica e informacional. // Acimed. 12:5 (Sept-Oct. 2004).

Ollé Castellà, C. (2010). Impacte de les revistes electròniques sobre la conducta informativa del personal docent i investigador de les universitats catalanes. Universitat de Barcelona. Departament de Biblioteconomia i Documentació, 2010. Tesis doctoral.

Ollé, C.; Borrego, A. (2010). A qualitative study of the impact of electronic journals on scholarly information behavior. // Library \& Information Science Research. 32:3 (Julio 2010) 221-228.

Pettigrew, K. E.; McKechnie, L. E. F. (2001). The use of theory in information science research. // Journal of the American Society for Information Science and Technology. 52:1 (2001) 62-73.

Pinto, M.; Fernández-Marcial, V.; Gómez-Camarero, C (2010). The Impact of Information Behavior in Academic Library Service Quality: A Case Study of the Science and Technology Area in Spain. // Journal of Academic Librarianship. 36:1 (2010) 70-78.

Puertas, S.; Pinto, M. (2009). El aprendizaje por competencias transversales: la competencia informacional y comunicacional de los estudiantes de la titulación de Comunicación Audiovisual. // Revista Académica de la Federación Latinoamericana de facultades de comunicación social. 78 (Enero-Julio 2009) 1-14.

Ramírez Velázquez, C. A. (2011). La satisfacción de las necesidades de información como factor de cambio en la identidad indígena de la comunidad amuzga: Nuevos avances. // Calva González, J. J. (coordinador). Investigaciones sobre las necesidades de información, el comportamiento informativo y la satisfacción en diferentes comunidades: proyectos, avances y resultados. México D. F.: Universidad Nacional Autónoma de Mexico, 2011.

Repanovici, A. y A. Landoy. (2007). La alfabetización informativa aplicada a los recursos electrónicosExperiencias de Brasov, Rumanía y Bergen, Noruega. // World Library and Information Congress: 76th IFLA Conference and Council. Durban, South Africa, 19-23 Agosto, 2007.

Rodríguez Cruz, Y.; Pinto Molina, M. (2010). Evolución, particularidades y carácter informacional de la toma de decisiones organizacionales. // Acimed. 21:1, 2010.

Romanos de Tiratel, S. (2000). Conducta informativa de los investigadores argentinos en Humanidades y Ciencias Sociales. // Revista Española de Documentación Científica. 23:3 (2000) 267-285.

Santos Rosas, A. (2009). La recuperación de información a través de los Sistemas de Información Geográfica. /I Calva González, J. J. (coordinador). La investigación sobre las necesidades de información en diferentes comunidades: Memoria del III Seminario de Usuarios de Información. México D. F.: UNAM, Centro Universitario de Investigaciones Bibliotecológicas (2009) 109-132.

Santos Rosas, A.; Calva González, J. J. (2008). El comportamiento informativo de los investigadores en geografía: el Instituto de Geografía de la UNAM. // Revista General de Información y Documentación. 18 (2008) 257-292. 
Sanz Casado, E. (1994). Manual de estudios de usuarios. Madrid: Fundación Germán Sánchez Ruipérez, 1994.

Saracevic, T. (2010). Information science. Bates, M. J.; Maack, M. N. (eds. ). Encyclopedia of Information Science. New York: Taylor and Francis, 2010.

Serrat Brustenga, M.; Sunyer Lázaro, S. (2008). El Centro de Recursos para el Aprendizaje y la Investigación (CRAI) en permanente transformación: servicios y recursos para el nuevo usuario 2. 0. // X Jornadas de Gestión de la Información, Madrid (Spain), 20-21 Noviembre 2008, 117-130.

Spink, A. (2010). Information behavior: An evolutionary instinct. Dordrecht: Springer Verlag, 2010.

Spink, A.; Cole, C. (2004). A human information behavior approach to a philosophy of information. // Library Trends. 52:3 (2004) 617-628.

Tannuri de Oliveira, E. F.; Castro Silva Casarin, H. de; Moreira García, R. (2007). El uso de la información científica en la producción de tesis de posgrado de la Universidade Rodrigo Moreira García Estadual Paulista, Marília. // Scire: representación y organización del conocimiento. 13:2 (2007) 129-137.

Uribe Tirado, A. (2008). Diseño, implementación y evaluación de una propuesta formativa en alfabetización informacional mediante un ambiente virtual de aprendizaje a nivel universitario: caso Escuela Interamericana de $\mathrm{Bi}$ bliotecología, Universidad de Antioquía. Universidad EAFIT, Medellín. 2008. Tesis de maestría.

Uribe Tirado, A. (2010). La alfabetización informacional en la universidad. Descripción y categorización según los niveles de integración de ALFIN. Caso Universidad de An- tioquia. // Revista Interamericana de Bibliotecología. 33:1 (2010) 31-83.

Vega Almeida, R. L.; Fernández Molina, J. C.; Linares Columbié, R. (2009). Coordenadas paradigmáticas, históricas y epistemológicas de la Ciencia de la información: una sistematización. // Information Research. 14:2 (2009) 14-2.

Villaseñor Rodríguez, I. (2009). Los estudios de usuarios publicados en España en el siglo XXI. // Calva González, J. J. (coordinador). La investigación sobre las necesidades de información en diferentes comunidades: Memoria del III Seminario de Usuarios de Información. México D. F.: UNAM, Centro Universitario de Investigaciones Bibliotecológicas (2009) 3-78.

Villaseñor Rodríguez, I. (2010a). Los estudios de usuarios de información como línea de investigación prioritaria en bibliotecología y documentación. Memoria del XXVII Coloquio de Investigación Bibliotecológica y sobre la Información: la investigación y la educación bibliotecológica en la sociedad del conocimiento. México D. F.: UNAM, Centro Universitario de Investigaciones Bibliotecológicas (2010) 161-175.

Villaseñor Rodríguez, I. (2010b). Aportaciones españolas a la teoría del fenómeno de las necesidades de información. // V Seminario de usuarios de la información: El fenómeno de las necesidades de información en diferentes comunidades. México D. F.: UNAM, Centro Universitario de Investigaciones Bibliotecológicas, 15-16 Abril 2010. 